$10.1117 / 2.1201405 .005483$

\title{
Silicon microcavity-based photovoltaic cells for IR harvesting
}

Moisés Garín, Roberto Fenollosa, Ramón Alcubilla, Lei Shi, Lluís F. Marsal, and Francisco Meseguer

Photovoltaic cells on silicon spherical microcavities trap IR light, increasing the probability of photon absorption and enhancing the efficiency of solar energy devices.

Photovoltaic (PV) cells are the primary device for converting sunlight directly into electricity. Most are made of silicon, a material relatively cheap to produce, and which has a reasonable ratio of efficiency to cost. However, widespread uptake of PV remains limited because, despite reductions in the price of solargrade silicon, the total cost of PV energy (in $\$ / \mathrm{kWh}$ ) remains high. Furthermore, commercial silicon-based PV cells have relatively low efficiency rates, at around $18 \%$ or less. To overcome this technological limitation, we would need to increase the efficiency of a PV cell while maintaining or even decreasing its processing costs.

One common approach to lowering costs is to reduce the quantity of silicon required by using thinner substrates, but this sacrifices efficiency. Instead, to maintain efficiency standards, we can use light-trapping techniques, where more light is absorbed in less silicon. For example, photodiode devices on microand nanophotonic structures enable confinement of incoming light inside photonic features, enhancing photocarrier generation. This phenomenon is seen in nanowire resonators, ${ }^{1}$ which demonstrate efficiency values beyond the Shockley-Queisser limit (the theoretical maximum efficiency of a cell). ${ }^{2,3}$ Simultaneously, photoexcited carriers are generated close to the collecting electrodes, boosting the cells' power generation capacity. ${ }^{1-4}$ However, because of the indirect nature of the bandgap in silicon (where the photoexcited electrons need to be pumped to the conduction band), photons with energy close to or below the bandgap $(\lambda>1150 \mathrm{~nm})$ produce negligible photoexcitation, and most IR light is lost.

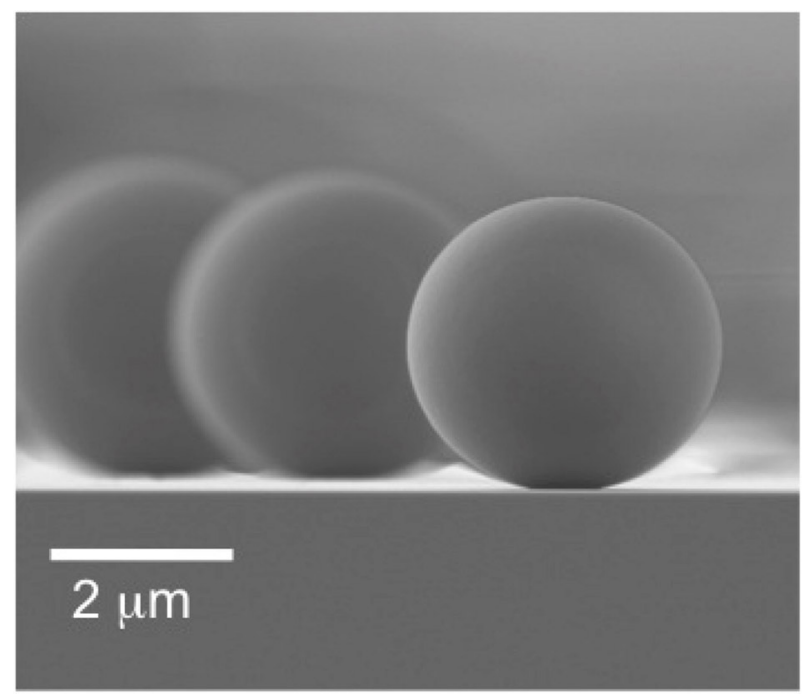

Figure 1. Scanning electron microscopy image of several silicon microcavities.

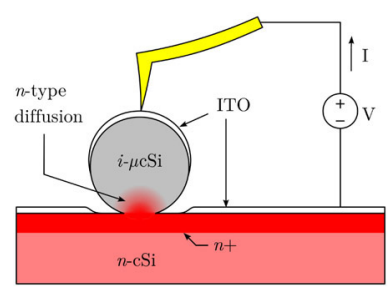

(a)

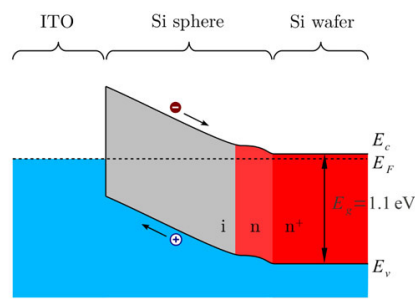

(b)
Figure 2. (a) Spherical device schematic. Back contact is made through the substrate, while front contact is achieved using a platinum atomic force microscope tip. (b) Band diagram proposal for the device. The direction of carrier separation is indicated for convenience. (Reprinted with permission. ${ }^{5}$ ) ITO: Indium tin oxide. Si: Silicon. cSi: Crystalline silicon. n: n-doped (silicon substrate). eV: Electron volt. $E_{c}, E_{F}, E_{V}$ : Electric field strengths. i: Insulator. 


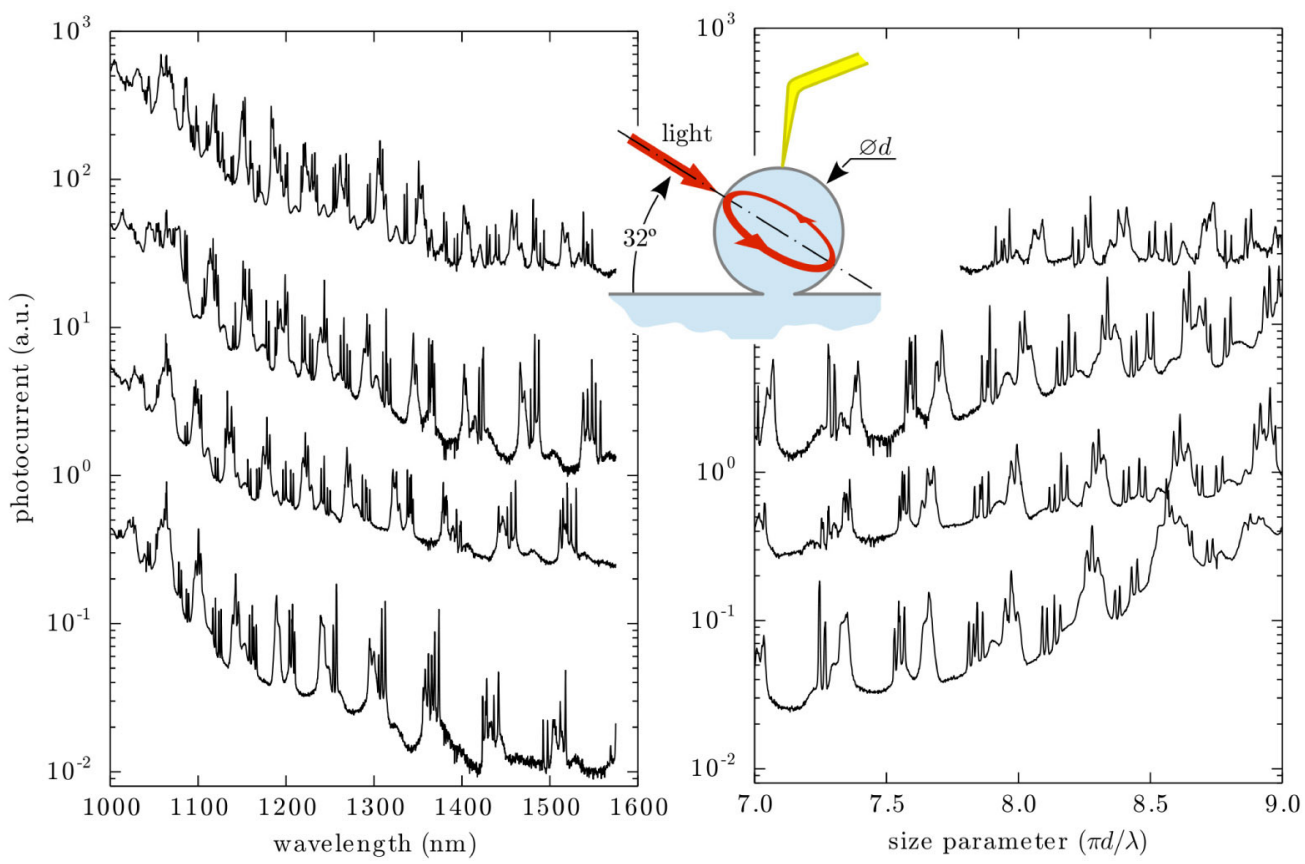

Figure 3. Left: Spectral response (short-circuit photocurrent) of microcavities with diameters of 3.9, 3.3, 3.2, and 2.9 $\mu m$, from top to bottom, respectively. Right: The same spectra but plotted as a function of the size parameter. In both cases the measured curves are shifted vertically for clarity. (Reprinted with permission. ${ }^{5}$ ) a.u.: Arbitrary units. ød: Diameter.

To overcome this bottleneck, we fabricated silicon photodiodes on spherical microcavities, ${ }^{5}$ which sustain well-defined Mie (or whispering gallery) modes $^{6}$ - those in which light is trapped in a sphere-in the optical region. These high- $Q$ resonant devices-where $Q$ is a measure of the light-trapping effect of the device-confine IR photons in the cavity for very long times, increasing the probability that they will be absorbed. For example, the light trapped in a Mie mode with $Q$-factor values, $Q \sim 6 \times 10^{3}$, for a typical wavelength of $1100 \mathrm{~nm}$, would remain in the microcavity for $3.5 \times 10^{-12}$ seconds, the time needed to travel a distance of $300 \mu \mathrm{m}$ in bulk silicon (the thickness of a standard silicon solar cell).

To develop rectifying junctions in the particles, we synthesized the silicon microcavities by chemical vapor deposition techniques ${ }^{6}$ directly onto $n$-type silicon substrates with an $n+$ layer implanted on top: see Figure 1 . We then annealed the samples at temperatures of between 850 and $1100^{\circ} \mathrm{C}$, allowing recrystallization of the microspheres and diffusion of $n$-type impurities from the substrate into the underside of the particles. Finally, we sputtered a very thin coating $(10 \mathrm{~nm})$ of indium tin oxide (ITO), and obtained thousands of ITO/sphere/n-Si devices, back-connected through the substrate. Figure 2(a) shows the basic structure of the device, where each sphere is contacted through a conductive atomic force microscope tip, while
Figure 2(b) shows a tentative band diagram assuming a PINlike diode structure (characterized by a semiconductor region between $\mathrm{p}$ - and n-type semiconductors). ${ }^{5}$ To record the spectral response of the devices, we focused a tunable monochromatic light source on the microcavity at an angle of $32^{\circ}$ to the plane, which minimized the influence of the substrate on the microcavity resonant modes. Figure 3 (left) shows the spectral response of the photocurrent in the near-IR region for different microcavities with diameters $3.9,3.3,3.2$, and $2.9 \mu \mathrm{m}$. The obtained photoresponse shows very rich spectra with plenty of high- $Q$ resonant peaks, ${ }^{6}$ which are about five times larger than the baseline. All these peaks correspond to the whispering gallery modes appearing in a spherical microcavity: see Figure 3 (right). ${ }^{5}$ We have obtained external quantum efficiency values (the ratio of charge carriers collected by a cell to the incoming photons) of around $1 \%$ at mode resonating wavelengths of up to $1500 \mathrm{~nm}$, a remarkable value for such a tiny single particle.

Our work takes a first step toward PV cells with a reasonable spectral response in the IR region where silicon shows negligible absorption. Due to the high refractive index of silicon, microspheres made of this material may be good candidates for highly scattering systems. ${ }^{7}$ A connected network of high

Continued on next page 
Q-factor microcavity-based PV cells, combining both resonant absorption and multiple scattering effects, might be the solution for efficient harvesting of IR sunlight. To that end, we are now developing a centimeter-size PV cell to incorporate in such a system.

\section{Author Information}

\section{Moisés Garín and Ramón Alcubilla}

Department of Electronic Engineering

Technical University of Catalunya

Barcelona, Spain

Moisés Garín holds a post-doctoral position with research interests in technology development for PV applications, focusing on metamaterial and nanophotonic approaches. His current research activities include wafer multiplication through macroporous silicon reorganization and silicon spherical microresonators applied to efficient light harvesting.

Ramón Alcubilla has worked in solar cell technology development for 20 years, undertaking pioneering work on surface passivation, laser processing, and multiple wafer splitting. He is currently a professor of electronics.

\section{Roberto Fenollosa and Francisco Meseguer}

Spanish National Research Council

Valencia, Spain

Roberto Fenollosa is a physicist currently researching the synthesis of silicon microspheres and their optical properties, as well as exploring technological applications from this material.

Francisco Meseguer is a research professor whose interests include colloid-based materials (photonic crystals and silicon colloids) and optical materials and metamaterials for light and sound.

\section{Lei Shi}

Department of Physics

Fudan University

Shanghai, China

Lei Shi is an associate professor whose research interests include light-matter interactions for technological applications. Examples are energy harvesting, new-generation light sources, and sensors. He has co-authored more than 33 journal papers.

\section{Lluís F. Marsal \\ Department of Electrical Engineering \\ Rovira i Virgili University \\ Tarragona, Spain}

Lluís F. Marsal is a professor with research interests in nanophotonics and sensor devices based on micronanoporous silicon and organic materials.

\section{References}

1. L. Cao et al., Engineering light absorption in semiconductor nanowire devices, Nat. Mater. 8, pp. 643-647, 2009.

2. J. Wallentin et al., InP nanowire array solar cells achieving $13.8 \%$ efficiency by exceeding the ray optics limit, Science 339, pp. 1057-1060, 2013.

3. P. Krogstrup et al., Single-nanowire solar cells beyond the Shockley-Queisser limit, Nat. Photon. 7, pp. 306-310, 2013

4. S. K. Kim et al., Tuning light absorption in core/shell silicon nanowire photovoltaic devices through morphological design, Nano Lett. 12, pp. 4971-4976, 2012.

5. M. Garín, R. Fenollosa, R. Alcubilla, L. F. Marsal, L. Shi, and F. Meseguer, Allsilicon spherical-Mie-resonator photodiode with spectral response in the infrared region Nat. Commun. (3440), 2014. doi:10.1038/ncomms4440

6. R. Fenollosa, F. Meseguer, and M. Tymczenko, Silicon colloids: from microcavities to photonic sponges, Adv. Mater. 20, pp. 95-98, 2008.

7. S. John, Strong localization of photons in certain disordered dielectric superlattices, Phys. Rev. Lett. 58, pp. 2486-2489, 1987. 